\title{
Indicadores preditivos dos volumes das secreções para a decanulação de pacientes em unidade de terapia intensiva
}

\author{
Predictive indicators of the volumes of the secretions for the decanulation of patients in the \\ intensive care unit \\ Indicadores predictivos de los volúmenes de las secreciones para la decanulación de pacientes en la \\ unidad de cuidados intensivos
}

\section{Resumo}

O objetivo deste trabalho foi o de estabelecer indicadores preditivos dos volumes das secreções para a decanulação de pacientes em unidade de terapia intensiva. Para mensurar a secreção foram elaboradas réguas adesivas para os vacuômetros e padronização para a aspiração com a medida de $10 \mathrm{ml}$ de soro fisiológico a $0,9 \%$ estilado durante o processo de aspiração e mais $10 \mathrm{ml}$ para a limpeza do sistema de aspiração, perfazendo um total de $20 \mathrm{ml}$ que foram deduzidos dos totais coletados na aspiração, tais critérios foram usados da mesma maneira em toda a amostra. Dos 52 $(59,77 \%)$ pacientes com taxa de secreção de 0 a $20 \mathrm{ml}$, apresentaram aspecto da secreção fluído, coloração clara e ausência de secreção na região da traqueostomia. Os parâmetros clínicos fonoaudiológicos evidenciaram: fonação e tempo de trânsito oral adequado e condições de manter o cuff desinsuflado. Tais resultados foram significativos para a decanulação ( $\mathrm{p}=0,000)$. Para $19(21,84 \%)$ pacientes com taxa de secreção entre 30 e 40ml, apresentaram aspecto de secreção espessa, coloração amarelada e presença de secreção na região da traqueostomia. Os parâmetros clínicos fonoaudiológicos evidenciaram: qualidade vocal disfônica, voz molhada, tempo de trânsito oral lento e dificuldade para manter o cuff desinsuflado. Tais resultados foram preponderantes para a não decanulação com significância ( $\mathrm{p}=$ $0,000)$ para todos os critérios. A análise dos volumes das secreções aspiradas, bem como o seu aspecto e coloração, evidenciaram ser um significativo indicador no processo de decanulação, à medida que quantifica e oferece padrões mensuráveis.

Palavras-chave: Traqueostomia; Taxa secretória; Transtornos de deglutição; Fonoaudiologia.

\begin{abstract}
The aim of this study was to establish predictive indicators of secretion volumes for decannulation in intensive care unit patients. To measure the secretion, adhesive rulers were prepared for the vacuometers and standardization for suction with the measurement of $10 \mathrm{ml}$ of saline solution at $0.9 \%$ styrated during the suction process and another $10 \mathrm{ml}$ for cleaning of the suction system, totaling $20 \mathrm{ml}$ that were deducted from the totals collected during suction, such criteria were used in the same way in the entire sample. Of the $52(59.77 \%)$ patients with secretion rate of 0 to $20 \mathrm{ml}$, the appearance of the secretion was fluid, clear color and absence of secretion in the tracheostomy region. The phonoaudiological clinical parameters showed: adequate phonation and oral transit time and conditions to maintain the cuff deflated. Such results were significant for decannulation $(\mathrm{p}=0.000)$. For $19(21.84 \%)$ patients with secretion rate between 30 and $40 \mathrm{ml}$, they presented the aspect of thick secretion, yellowish color and presence of secretion in
\end{abstract}


the tracheostomy region. The phonoaudiological clinical parameters showed: dysphonic vocal quality, wet voice, slow oral transit time and difficulty in maintaining the cuff deflated. Such results were preponderant for nondecannulation with significance $(\mathrm{p}=0.000)$ for all criteria. The analysis of the volume of suctioned secretions, as well as their aspect and coloration, proved to be a significant indicator in the decannulation process, as it quantifies and offers measurable standards.

Keywords: Tracheostomy; Secretory rate; Swallowing disorders; Speech therapy.

\section{Resumen}

El objetivo de este trabajo fue establecer indicadores predictivos de los volúmenes de secreción para la decanulación en pacientes de la unidad de cuidados intensivos. Para la medición de la secreción se prepararon reglas adhesivas para los vacuómetros y la estandarización para la succión con la medición de $10 \mathrm{ml}$ de solución salina al $0,9 \%$ de estireno durante el proceso de succión y otros $10 \mathrm{ml}$ para la limpieza del sistema de succión, totalizando $20 \mathrm{ml}$ que fueron descontados de los totales recogidos durante la succión, dichos criterios fueron utilizados de la misma manera en toda la muestra. De los $52(59,77 \%)$ pacientes con un índice de secreción de 0 a $20 \mathrm{ml}$, el aspecto de la secreción era fluido, de color claro y con ausencia de secreción en la región de la traqueotomía. Los parámetros clínicos fonoaudiológicos mostraron: tiempo de fonación y tránsito oral adecuados y condiciones para mantener el manguito desinflado. Estos resultados fueron significativos para la decanulación ( $\mathrm{p}=0,000)$. Para $19(21,84 \%)$ pacientes con

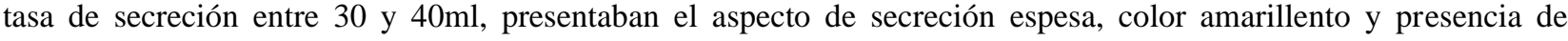
secreción en la región de la traqueostomía. Los parámetros clínicos fonoaudiológicos mostraron: calidad vocal disfónica, voz húmeda, tiempo de tránsito oral lento y dificultad para mantener el manguito desinflado. Dichos resultados fueron preponderantes en el caso de la no desconexión, con significación ( $\mathrm{p}=0,000)$ para todos los criterios. El análisis del volumen de las secreciones aspiradas, así como su aspecto y coloración, resultó ser un indicador importante en el proceso de decanulación, ya que cuantifica y ofrece estándares medibles.

Palabras clave: Traqueostomía; Tasa de secreción; Trastornos de la deglución; Logopedia.

\section{Introdução}

A traqueostomia é um procedimento cirúrgico realizado com frequência nas Unidades de Terapia Intensiva com a finalidade de promover uma via aérea artificial prolongada. Calcula-se que aproximadamente $40 \%$ dos pacientes da Unidade de Terapia Intensiva (UTI) encontram-se em ventilação mecânica (VM), (Nelson et al., 2010; Cunha et al., 2012) destes aproximadamente 10 a 24\% são dependentes de VM por períodos prolongados, e são submetidos à traqueostomia (TQT), sendo considerada uma alternativa para o uso prolongado de VM, na facilitação para a remoção das secreções traqueobrônquicas, redução do risco de pneumonia associada à VM e a boncoaspiração de secreções orais ou gástricas com o fechamento da traqueia pela insuflação do balonete (Ferreira et al., 2012; Ibrahim et al., 2012; Mallmann, 2019).

Após a estabilidade dos parâmetros respiratórios é iniciada a retirada da VM, período em que o paciente passa pela readaptação da autonomia respiratória, com pouco ou nenhum suporte ventilatório (Albuquerque, 2011; De Mestral et al., 2011; Azevedo et al., 2013; Malgaço et al., 2021).

Apesar de todos os cuidados e critérios utilizados durante os procedimentos de manejo da TQT, o momento mais sensível, ainda, é a sua descontinuação (período pelo qual o paciente passa da situação de dependência da TQT para uma situação de independência), sendo posteriormente realizada a decanulação (Clini et al., 2011; Lemos et al., 2019), porém a precocidade dessa remoção pode relacionar-se a riscos como: a dificuldade de acesso às vias aéreas, prejuízo na troca gasosa, broncoaspiração e aumento da ocorrência de pneumonia ocasionando uma alta taxa de mortalidade (Albuquerque, 2011; Côrte, Vicente e Friche, 2019).

Contudo, para que este processo ocorra de maneira segura é necessária à observância de alguns biomarcadores como: estabilidade hemodinâmica, ausência ou diminuição da secreção pulmonar, melhora do quadro respiratório, ausência de sinais de esforços respiratórios, boa saturação de O2, ausência da necessidade de ventilação mecânica, tosse eficaz, deglutição adequada, presença de eupneia e bons resultados em exames laboratoriais: hemograma, eletrólitos e gasometria (Dantas et al. 2010; Frengley, 2014; Mallmann, 2019).

O aspecto e a quantidade de secreção estão associados com a piora no quadro de evolução do paciente, ocasionando o insucesso da decanulação fator que alargaria o tempo de permanência na UTI, ocasionando o aumento da morbidade e 
mortalidade. Não obstante, quanto se trata da mensuração da secreção não há estudo na literatura que estabeleçam biomarcadores relacionados à quantidade e aspecto e suas exatas influências na decanulação (Santana, 2014; Furkim et al., 2014).

Entretanto, uma melhor compreensão desta correlação poderá contribuir no planejamento de condutas, que visem à prevenção de complicações relacionadas à decanulação, bem como o aperfeiçoamento das estratégias no processo de avaliação e manejo dos pacientes traqueostomizados.

Assim, o objetivo desse estudo foi analisar os volumes e aspectos das secreções aspiradas de pacientes traqueostomizados e internados em UTI, submetidos à cirurgia de revascularização miocárdica, a fim de detectar a influência desta mensuração no processo de decanulação da traqueostomia.

\section{Metodologia}

Trata-se de um estudo observacional analítico de delineamento de coorte, realizado em duas UTI Cardiológicas do Hospital Santa Casa de Misericórdia de Curitiba, sendo composta por 87 pacientes com diagnóstico de doença cardíaca, submetidos à revascularização miocárdica, e por consequência de complicações intra-operatório ou pós-operatório tiveram a necessidade do uso prolongado de ventilação mecânica (VM) que culminou na realização da traqueostomia.

Fizeram parte deste estudo 51 pacientes do sexo masculino e 36 do sexo feminino, com média de idade de 68,57 anos (desvio padrão de 9,28). Os critérios de inclusão foram: pacientes adultos com diagnóstico de doença cardíaca e submetidos à revascularização miocárdica eletiva; traqueostomizados no mínimo há sete dias; estabilidade hemodinâmica; saturação de SpO2 estável há > 24h em ar ambiente; radiografia do tórax sem alterações; apresentar respiração espontânea e ausência de desconforto respiratório (Presto \& Damázio, 2009; David \& Godwasser, 2011; Medeiros et al., 2019).

Os critérios de exclusão foram: presença de história ou sinais sugestivos de obstrução de vias aéreas ou de doenças respiratórias; quadro séptico ativo; nível de consciência rebaixado com Glasgow Coma Score persistentemente menor que oito pontos; doença de base neurológica; instabilidade hemodinâmica; critério clínico de manutenção da via aérea artificial; a não tolerância ao teste de respiração espontânea; disfagia importante com aspirações frequentes (David \& Godwasser, 2011; Mallmann, 2019).

Para mensurar a secreção foram elaboradas três réguas adesivas para diferentes marcas de vacuômetros: Protec®, Venturi®, Moriya®. Após a confecção das réguas, estas foram afixadas nos respectivos vacuômetros e estabelecida com a equipe de fisioterapeutas a padronização para a aspiração, com a medida de $10 \mathrm{ml}$ de soro fisiológico a 0,9\% estilado durante o processo de aspiração e mais $10 \mathrm{ml}$ para a limpeza do sistema de aspiração, perfazendo um total de $20 \mathrm{ml}$ que foram deduzidos dos totais coletados na aspiração, tais critérios foram usados da mesma maneira em toda a amostra. Após a realização das avaliações, os pacientes foram divididos em dois grupos, para análises posteriores.

O primeiro, composto por pacientes que apresentaram condições adequadas para decanular. O segundo grupo foi constituído por pacientes que por complicações durante o processo de decanulação, não puderam ser decanulados. De ambos os grupos foi realizada a análise descritiva das variáveis relacionadas aos dados clínicos e de condições para a decanulação, comparados com o volume e aspecto das secreções aspiradas destes pacientes.

Os procedimentos estatísticos utilizados neste estudo foram compostos de análise descritiva e inferencial (teste de significância), cálculo da média e desvio padrão, que foi aplicado em todas as tabelas. Assim como, para a análise estatística aplicou-se o Teste Qui-quadrado, constituindo a base do processo comparativo das distribuições dos dados tabelados referentes à relação dos achados dos volumes das secreções aspiradas das vias aéreas com os parâmetros de avaliação para a decanulação, tendo para o nível de significância de $\mathrm{p}<0,05$ e poder do teste de $90 \%$. 
Todos os pacientes assinaram do Termo de Consentimento Livre e Esclarecido, além da devida autorização do médico responsável da UTI. Esta pesquisa foi submetida e aprovada no CONEP sob o nº 146.315. CAAE: 09142812.2.0000.0103.

\section{Resultados}

Os resultados da amostra dos volumes das secreções mensuradas foram dispostos segundo a análise da relação entre condições de decanular, taxa de secreção e os parâmetros de avaliação para decanulação. As taxas de secreção foram pareadas para melhor análise estatística da seguinte forma 0 a $20 \mathrm{ml}$ e 30 a $40 \mathrm{ml}$.

Dos 87 pacientes, 52 decanulados, $20(38,46 \%)$ não apresentaram secreção e $32(61,54 \%)$ apresentaram taxa de secreção até $20 \mathrm{ml}$, com proporção significativamente $(\mathrm{p}=0,000)$ para o aspecto fluído $30(93,75 \%)$ contra $2(6,25 \%)$ espessa.

Os 35 pacientes com taxa de secreção entre 30 e 40ml, não puderam ser decanulados dos quais 28 (80\%) apresentaram significância $(\mathrm{p}=0,000)$ para o aspecto da secreção espessa, e apenas $7(20 \%)$ para o aspecto de secreção fluída, evidenciando a relação direta entre taxa de secreção e o aspecto da secreção.

Tabela 1: Relação entre condições de decanular, taxa e aspecto da secreção.

\begin{tabular}{|c|c|c|c|c|c|c|}
\hline \multirow{2}{*}{$\begin{array}{l}\text { Condições de } \\
\text { decanular }\end{array}$} & \multirow{2}{*}{$\begin{array}{c}\text { Taxa de } \\
\text { Secreção }\end{array}$} & \multicolumn{5}{|c|}{ Aspecto da Secreção } \\
\hline & & Sem secreção & Espessa & Fluída & TOTAL & Valor de $\mathrm{p}$ \\
\hline \multirow[b]{2}{*}{ SIM } & $0 \mathrm{ml} \mathrm{a} 20 \mathrm{ml}$ & 20 & 2 & 30 & 52 & 0,000 \\
\hline & $30 \mathrm{ml} \mathrm{a} 40 \mathrm{ml}$ & 0 & 0 & 0 & 0 & \\
\hline \multirow[t]{2}{*}{ NÃO } & $0 \mathrm{ml} \mathrm{a} 20 \mathrm{ml}$ & 0 & 0 & 0 & 0 & \\
\hline & $30 \mathrm{ml} \mathrm{a} 40 \mathrm{ml}$ & 0 & 28 & 7 & 35 & 0,000 \\
\hline TOTAL & & 20 & 30 & 37 & 87 & \\
\hline
\end{tabular}

1Valor de p obtido pela comparação entre secreção espessa e fluída. ${ }^{2}$ Teste Qui-quadrado ao nível de significância de 0,05 Fonte: Autores (2021).

Dos pacientes decanulados, $30(93,75 \%)$ apresentaram coloração clara e apenas $2(6,25 \%)$ coloração amarelada para uma taxa de secreção até $20 \mathrm{ml}$ com relação de significância de $(\mathrm{p}=0,000)$ para condições de decanular. Contudo os pacientes que não puderam ser decanulados foram significativas em $23(76,67 \%)$ para a secreção de coloração amarelada e taxa de secreção de 30 a 40ml (p = 0,000). $74(85,06 \%)$ pacientes tiveram condições de manter o cuff desinsuflado, sendo a maior ocorrência para aqueles que apresentaram taxa de secreção até 20ml em 52 (70,27\%) dos casos, contra 22 (29,73\%) com taxa de secreção igual ou superior a 30ml e não puderam decanular. Verificou-se ainda a existência de relação significativa (p $=$ 0,000) entre as taxas de secreção, manutenção do cuff desinsuflado e condições de decanular. 
Tabela 2: Relação entre condições de decanular, taxa e coloração da secreção.

\begin{tabular}{|c|c|c|c|c|c|c|c|}
\hline \multirow{2}{*}{$\begin{array}{l}\text { Condições de } \\
\text { decanular }\end{array}$} & \multirow{2}{*}{$\begin{array}{r}\text { Taxa de } \\
\text { Secreção }\end{array}$} & \multicolumn{5}{|c|}{ Coloração da Secreção } & \multirow[b]{2}{*}{ Valor de $p$} \\
\hline & & $\mathbf{I}$ & II & III & IV & TOTAL & \\
\hline \multirow{2}{*}{ SIM } & $0 \mathrm{ml} \mathrm{a} 20 \mathrm{ml}$ & 20 & 30 & 2 & 1 & 53 & 0,000 \\
\hline & $30 \mathrm{ml} \mathrm{a} 40 \mathrm{ml}$ & 0 & 0 & 0 & 0 & 0 & \\
\hline \multirow[t]{2}{*}{ NÃO } & $0 \mathrm{ml}$ a $20 \mathrm{ml}$ & 0 & 0 & 0 & 0 & 0 & \\
\hline & $30 \mathrm{ml} \mathrm{a} 40 \mathrm{ml}$ & 0 & 7 & 23 & 4 & 34 & 0,000 \\
\hline TOTAL & & 20 & 37 & 25 & 5 & 87 & \\
\hline
\end{tabular}

Legenda: I - Sem secreção; II - Secreção Clara; III - Secreção amarelada; IV - Secreção sanguinolenta. ${ }^{1}$ Teste Qui-quadrado ao nível de significância de 0,05 .

Fonte: Autores (2021).

Quanto aos achados da secreção na região da traqueostomia, foi verificado a presença em 5 (9,92\%) e ausência em 47 $(90,38 \%)$ dos casos para uma taxa de secreção até 20ml, com significância estatística $(\mathrm{p}=0,000)$ para condições de decanular. Dos pacientes que apresentaram taxa de secreção igual ou superior a 30ml foram encontradas a presença de secreção na região da traqueostomia em $22(62,86 \%)$ e ausência em 13 (37,14\%) dos casos, sendo que os pacientes que apresentaram secreção em região da traqueostomia não puderam ser decanulados $(\mathrm{p}=0,000)$.

Tabela 3: Condições de decanular, taxa de secreção, condição de manter o cuff desinsuflado e presença de secreção em TQT.

\begin{tabular}{|c|c|c|c|c|c|c|c|}
\hline \multirow{2}{*}{$\begin{array}{c}\text { Condições de } \\
\text { decanular }\end{array}$} & \multirow{2}{*}{$\begin{array}{c}\text { Taxa de } \\
\text { Secreção }\end{array}$} & \multicolumn{3}{|c|}{ Manter o cuff desinsuflado } & \multicolumn{3}{|c|}{ Presença de secreção em TQT } \\
\hline & & Sim & Não & TOTAL & Presente & Ausente & TOTAL \\
\hline \multirow{2}{*}{ SIM } & $0 \mathrm{ml} \mathrm{a} 20 \mathrm{ml}$ & 52 & 0 & 52 & 5 & 47 & 52 \\
\hline & $30 \mathrm{ml} \mathrm{a} 40 \mathrm{ml}$ & 0 & 0 & 0 & 0 & 0 & 0 \\
\hline \multirow{2}{*}{ NÃO } & $0 \mathrm{ml} \mathrm{a} 20 \mathrm{ml}$ & 0 & 0 & 0 & 0 & 0 & 0 \\
\hline & $30 \mathrm{ml} \mathrm{a} 40 \mathrm{ml}$ & 22 & 13 & 35 & 22 & 13 & 35 \\
\hline TOTAL & & 74 & 13 & 87 & 27 & 60 & 87 \\
\hline
\end{tabular}

1Valor de p obtido pela comparação entre condições ou não de manter o cuff desinsuflado e presença ou ausência de secreção em TQT.

2Teste Qui-quadrado ao nível de significância de 0,05 .

Fonte: Autores (2021).

Para a relação entre condições de decanular, taxa de secreção e o tempo de trânsito oral, foi observada a direta correlação entre tempo de trânsito oral adequado $46(88,46 \%)$ e lento $6(11,54 \%)(p=0,000)$ para uma taxa de secreção até 20ml. No entanto, para uma taxa de secreção de 30 a 40ml, apenas $5(14,29 \%)$ pacientes apresentaram tempo de trânsito adequado, contra $30(85,71 \%)(\mathrm{p}=0,0000)$ que tiveram o tempo de trânsito oral lento. Verificou-se a existência de relação significativa entre o tempo de trânsito oral, taxa de secreção e condições de decanular. 
Tabela 4: Relação entre condições de decanular, taxa de secreção e o tempo de trânsito oral.

\begin{tabular}{|c|c|c|c|c|c|}
\hline \multirow{2}{*}{$\begin{array}{c}\text { Condições de } \\
\text { decanular }\end{array}$} & \multirow{2}{*}{ Taxa de Secreção } & \multicolumn{4}{|c|}{ Tempo de trânsito oral } \\
\hline & & Adequado & Lento & TOTAL & Valor de $\mathrm{p}$ \\
\hline \multirow{2}{*}{ SIM } & $0 \mathrm{ml} \mathrm{a} 20 \mathrm{ml}$ & 46 & 6 & 52 & 0,000 \\
\hline & $30 \mathrm{ml} \mathrm{a} 40 \mathrm{ml}$ & 0 & 0 & 0 & \\
\hline \multirow[t]{2}{*}{ NÃO } & $0 \mathrm{ml} \mathrm{a} 20 \mathrm{ml}$ & 0 & 0 & 0 & \\
\hline & $30 \mathrm{ml} \mathrm{a} 40 \mathrm{ml}$ & 5 & 30 & 35 & 0,000 \\
\hline TOTAL & & 51 & 36 & 87 & \\
\hline
\end{tabular}

1Valor de p obtido pela comparação entre taxa de secreção e tempo de trânsito oral. ${ }^{2}$ Teste Qui-quadrado ao nível de significância de 0,05 . Fonte: Autores (2021).

No tocante a fonação em $44(84,62 \%)$ dos casos apresentaram fonação adequada, contra $8(15,38 \%)$ para disfonia e taxa de secreção de 0 a $20 \mathrm{ml}(\mathrm{p}=0,000)$. Todavia do total dos 39 pacientes que apresentaram taxa de secreção de 30 a $40 \mathrm{ml}$, $36(92,31 \%)$ foi verificado a presença de uma ou ambas alterações na fonação, sendo disfonia $19(52,78 \%)$ e 17 (47,22\%) voz molhada, não apresentaram êxito para a decanulação com significância de $(\mathrm{p}=0,0000)$. O resultado total da amostra teve uma frequência de 91 respostas, devido aos pacientes que apresentaram concomitantemente disfonia e voz molhada.

Tabela 5: Relação entre condições de decanular, taxa de secreção e fonação.

\begin{tabular}{|c|c|c|c|c|c|c|}
\hline \multirow{2}{*}{$\begin{array}{c}\text { Condições de } \\
\text { decanular }\end{array}$} & \multirow{2}{*}{$\begin{array}{c}\text { Taxa de } \\
\text { Secreção }\end{array}$} & \multicolumn{4}{|c|}{ Fonação } & \multirow[b]{2}{*}{ Valor de $\mathrm{p}$} \\
\hline & & Adequada & Disfônica & $\begin{array}{c}\text { Voz } \\
\text { molhada }\end{array}$ & TOTAL & \\
\hline \multirow{2}{*}{ SIM } & $0 \mathrm{ml} \mathrm{a} 20 \mathrm{ml}$ & 44 & 8 & 0 & 52 & \multirow[t]{2}{*}{0,000} \\
\hline & $30 \mathrm{ml} \mathrm{a} 40 \mathrm{ml}$ & 0 & 0 & 0 & 0 & \\
\hline \multirow{2}{*}{ NÃO } & $0 \mathrm{ml}$ a $20 \mathrm{ml}$ & 0 & 0 & 0 & 0 & \multirow{3}{*}{0,000} \\
\hline & $30 \mathrm{ml} \mathrm{a} 40 \mathrm{ml}$ & 3 & 19 & 17 & 39 & \\
\hline TOTAL & & 47 & 27 & 17 & 91 & \\
\hline
\end{tabular}

1Valor de p obtido pela comparação entre taxa de secreção e fonação. ${ }^{2}$ Teste Qui-quadrado ao nível de significância de 0,05 . Fonte: Autores (2021).

Portanto ao analisarmos de maneira geral os volumes das secreções aspiradas demonstraram que $52(59,77 \%)$ dos pacientes com taxa de secreção de 0 a 20ml, apresentaram aspecto da secreção fluída, coloração clara e ausência de secreção na região da traqueostomia. Em relação aos parâmetros clínicos fonoaudiológicos foram observados: fonação e tempo de trânsito oral adequado e condições de manter o cuff. Tais resultados foram preponderantes para a decanulação ( $\mathrm{p}=0,000$ ). Porém dos $19(21,84 \%)$ pacientes com taxa de secreção entre 30 a 40ml apresentaram aspecto de secreção espessa, de coloração amarelada, presença de secreção na região da traqueostomia.

Em relação aos parâmetros clínicos fonoaudiológicos foram observados: qualidade vocal disfônica, voz molhada, tempo de trânsito oral lento e dificuldade para manter o cuff desinsuflado. Tais resultados foram preponderantes para a não decanulação $(\mathrm{p}=0,000)$ para todos os critérios. 


\section{Discussão}

O acúmulo de secreção pulmonar pode provocar aumento da resistência das vias aéreas, obstrução parcial ou total das mesmas com consequente, hipoventilação alveolar e desenvolvimento de atelectasias, hipoxemia e aumento do trabalho respiratório. Além disso, a presença de estase de secreção e atelectasias constituem um meio favorável para o desenvolvimento de colonização bacteriana e infecção do parênquima pulmonar (David \& Godwasser, 2011; Mallmann, 2019).

Dessa forma, observa-se que a presença de secreção pulmonar constitui um fator complicador grave que pode prolongar o tempo de VMI e, consequentemente, afetar negativamente o prognóstico de sucesso na decanulação. Convém ressaltar que os pacientes internados nas UTIs cardíacas são, em sua maioria, idosos e, por sua vez, propensos a apresentar diversas comorbidades. Em um estudo multicêntrico que comparou o fator idade avançada com as comorbidades em pacientes com possibilidade de decanulação, evidenciou que o desmame ventilatório bem sucedido diminuiu os riscos de morte em $62 \%$ dos casos e os preditores de sucesso deste desmame, estão diretamente relacionados às comorbidades e as condições respiratórias, sendo que o fator idade não foi determinante para o desfecho dos resultados (Frengley, 2014; Lemos et al., 2019).

Quanto à presença de secreção na região da traqueostomia foi observada uma relação direta com a taxa de secreção aspirada, uma vez que 47 (90,38\%) não foi observada a presença de secreção da região da traqueostomia, para uma taxa de secreção aspirada de no máximo $20 \mathrm{ml}(\mathrm{p}=0,000)$, com condições de manter o cuff desinsuflado. Quanto ao aspecto da secreção, e a coloração como preditores do sucesso para decanulação, os resultados revelaram que os pacientes que apresentaram taxas de secreção até $20 \mathrm{ml}$, com o aspecto fluído e coloração clara, tiveram condições significativas ( $\mathrm{p}=0,000$ ) para decanular. Estes resultados evidenciaram a relação direta entre taxa de secreção, o aspecto e a coloração da secreção, revelando ser um dos fatores preponderantes no processo de decanulação. Muitos autores relatam a importância da secreção como critérios de avaliação para decanulação, destacando a não apresentação de secreção pulmonar em quantidade significativa a ponto de comprometer o padrão respiratório, resolução da origem do problema respiratório que ocasionaria a significativa produção de secreção ou ainda, pacientes que apresentem melhor manejo das secreções tem melhores condições para decanular (Nelson et al., 2010; Kutsukutsa et al., 2014; Côrte, 2019).

Outros autores realizaram estudos com objetivo de caracterizar e definir fatores que influenciam na decanulação e os resultados demonstraram que os critérios determinantes estão relacionados ao nível de consciência do paciente, a eficácia da tosse, a quantidade de secreções e a oxigenação (Padovani et al., 2013; Medeiros et al., 2019; Malgaço et al., 2021). Para tanto a secreção é um fator importante na decanulação, porém, não foi observado nenhum estudo que mensurasse a secreção de pacientes traqueostomizado em processo de decanulação. Os resultados referentes à fonação e intensidade vocal demonstraram que pacientes com maiores quantidades de secreção aspiradas também apresentaram alterações vocais como disfonia, voz molhada e intensidade fraca $(\mathrm{p}=0,000)$ não puderam ser decanulados.

A qualidade vocal depende das modificações realizadas em todo o trato vocal, sendo a voz molhada um termo que descreve o som borbulhante produzido na fonação e que indica estase de secreção e/ou saliva, no vestíbulo laríngeo, nas pregas vocais ou em todo o trato vocal, estas características estão frequentemente associadas ao aumento do risco de aspiração, já as alterações na intensidade vocal estão relacionadas à redução na amplitude, assim como no tempo de elevação laríngea que podem impactar na deglutição, com estase principalmente na região da transição faringoesofágica. Muitos autores afirmam que tais achados estão diretamente relacionados à diminuição da sensibilidade laríngea, que comprometeria a proteção das vias aéreas e por sua vez, ocasionaria no insucesso da decanulação (Furkim et al., 2014; Zanata et al. 2016; Côrte, 2019).

$\mathrm{Na}$ dinâmica da deglutição os resultados evidenciaram que os sujeitos com taxa de secreção igual ou maior que 30ml, apresentaram tempo de trânsito oral lento, elevação laríngea reduzida com múltipla deglutição, tosse durante a deglutição e ausculta cervical alterada antes e após a deglutição, além da presença de corante azul nas secreções aspiradas. Os achados deste estudo corroboram com autores que destacam que a traqueostomia altera a anatomia e a fisiologia do sistema 
respiratório, influenciando nos mecanismos de proteção das vias aéreas, da produção vocal, na dinâmica da deglutição, dificultando o movimento de elevação e anteriorização da laringe, por uma combinação de fatores que promovem a perda do mecanismo do reflexo glótico como a dessensibilização laringo-faríngea, a ausência da pressão subglótica, o menor tempo de fechamento das pregas vocais e a compressão do esôfago (Santana et al., 2014; Mandaville et al., 2014; Jung et al., 2018).

Outro fator é a relação do aspecto mecânico de limpeza da laringe com o fluxo aéreo, pois após o movimento de deglutição normal, ocorre um fluxo aéreo expiratório abrupto através das pregas vocais, para expelir eventuais restos alimentares que possam permanecer na região da laringe supraglótica e do espaço glótico. Nos pacientes traqueostomizados, esse mecanismo não ocorre, pois o ar expirado é desviado através da cânula de traqueostomia e não passa pelas pregas vocais, o que provocaria alterações neurofisiológicas de dessensibilização da laringe e das pregas vocais, visto que o ar é um constante estímulo para os mecanismos protetivos das vias aéreas (Medeiros et al., 2019). Contudo, a presença de traqueostomia é um complicador significativo e sua repercussão é imediata, desencadeando modificações na integração das funções respiratórias e de deglutição, comprometendo as funções motoras e sensoriais dos mecanismos de deglutição, além de complicações tardias como estenose traqueal, sangramento, fístulas, infecções, hemorragias e broncoaspiração (Garuti et al., 2014; Costa, 2016; Mallmann, 2019; Malgaço et al., 2021).

Para tanto os resultados expressos nesta discussão apontam que a quantidade, coloração e aspecto da secreção são fatores prováveis e preditores de sucesso na decanulação, haja vista a discrepância na média dos seus valores comparativos entre condições de decanular e taxa de secreção, sendo significativamente menor no grupo de sucesso que apontaram taxas de secreções $\leq 20 \mathrm{ml}$.

\section{Conclusão}

A análise dos volumes das secreções aspiradas, bem como sua coloração e aspecto, evidenciaram ser um significativo indicador no processo de decanulação de pacientes hospitalizados em unidades de terapia intensiva cardiológica, à medida que quantifica e oferece padrões mensuráveis para melhor atender aos requisitos de eficiência e segurança neste procedimento. Por ser um notório fator preditivo de influência no sucesso da decanulação, mas não o único, deve ser tomado como mais um marcador a ser levado em conta na avaliação durante todo o processo de decanulação da traqueostomia.

Contudo, os dados desta pesquisa são inéditos e colaboram com as futuras investigações sobre o desmame e a decanulação da traqueostomia em unidade de terapia intensiva.

Para pesquisas futuras, sugere-se investigar a mensuração da secreção de pacientes traqueostomizados com outras doenças de base, ou ainda o levantamento e acompanhamento da taxa de secreção de pacientes que necessitem de suporte ventilatório, fator este que poderia influenciar no desmame da ventilação mecânica.

\section{Agradecimentos}

Agradecemos ao Dr. Ricardo Gustavo Zill Risson, Médico Intensivista e Coordenador-geral das UTIs em que esta pesquisa foi realizada, ao Departamento de Mestrado e Doutorado da Universidade Tuiuti do Paraná, ao Hospital Santa Casa de Misericórdia de Curitiba e aos pacientes que participaram deste estudo.

\section{Referências}

Albuquerque, C. Ventilação mecânica e disfagia. In: Davi CM. Ventilação mecânica: da fisiologia à prática clínica. 2 ed. Rio de Janeiro: Revinter, 2011.

Azevedo, L. C., Park, M., Salluh, J. I., Rea-Neto, A., Souza-Dantas, V. C. et al. The ERICC (Epidemiology of Respiratory Insufficiency in Critical Care) investigators. Clinical outcomes of patients requiring ventilatory support in Brazilian intensive care units: a multicenter, prospective, cohort study. Crit Care. $2013 ; 17(2):$ R63. 
Beccaria, L. M., Pereira, R. A. M., Contrin, L. M., Lobo, S. M. A., Trajano, D. H. L. Eventos adversos na assistência de enfermagem em uma unidade de terápia intensiva. Revista Brasileira de Terapia Intensiva. São Paulo. 2009; 21(3): 276-82.

Clini, E. M., et al. Functional recovery following physical training in tracheotomized and chroni- cally ventilated patients.Respir Care. 2011 ; 56(3):306-13.

Côrte M. M. D., Vicente L. C. C., Friche A. A. L. Decanulação: indicadores sociodemográficos, clínicos e fonoaudiológicos preditivos de sucesso. Audiol Commun Res. 2019; 24:e2103.

Costa, C. C., Favero, T. C., Rosa, F. B., Steidl, E. M. S., Mancopes, R. Decanulação: atuação fonoaudiológica e fisioterapêutica. Distúrbios Comun. São Paulo, março, 2016,28(1): 93-101.

Cunha, M., Barosa, J., Margalho, P., Tomé, P., Laíns, J. Protocolo de Encerramento de Traqueotomia em Internamento em Reabilitação. Revista da Sociedade Portuguesa de Medicina Física e de Reabilitação. 2012; vol. 22, n. 2, ano 20, p. 28-35.

Dantas, M. O. R. L., Auler Jr, J. O. C., Andrade, C. R. F. Avaliação da deglutição de idosos com indicação de revascularização miocárdica. Pró-Fono Revista de Atualização Científica. 2010 out-dez;22(4):385-90.

David, C. M. N., Godwasser, R. Vias aéreas artificiais: intubação e traqueostomia. In: David CMN. Ventilação mecânica: da fisiologia à prática clínica. 2 ed. Rio de Janeiro: Revinter, 2011.

De Mestral, C., Iqbal, S., Fong, N., LeBlanc, J., et al., Impact of a specialized multidisciplinary tracheostomy team on tracheostomy care in critically ill patients. Can J Surg. 2011.Jun; 54(3):167-72.

Dias, C. M., Siqueira, T. M., Faccio, T. R., Gontijo, L. C., Salge, J. A. S. B., Volpe, M. S. Efetividade e segurança da técnica de higiene brônquica: hiperinsuflação manual com compressão torácica. Rev. Bras. Intensiva. 2011; 23(2):190-198.

Ferreira, V. M., Silva, B. C. M., Miranda, L. C., Reis, H. F. C. Perfil da utilização dos parâmetros de desmame: um estudo de revisão. EFDeportes.com, Revista Digital. Buenos Aires, Año 17, No 173, Octubre de 2012.

Frengley, J. D., Sansone, G. R., Shakya, K., Kaner, R. J. Prolonged Mechanical Ventilation in 540 Seriously Ill Older Adults: Effects of Increasing Age on Clinical Outcomes and Survival. Journal The American Geriatrics Society, Vol. 62, n. 1, January, 2014. 62:1-9.

Furkim, A. M., Barata, L., Duarte, S. T., Nascimento Junior, J. R. Gerenciamento fonoaudiológico da disfagia no paciente crítico na unidade de terapia intensiva. In: Furkim A M, Rodrigues KA. Disfagias nas unidades de terapias intensiva. São Paulo: Editora Roca; 2014. p. 111-126.

Garrubba, M., Turner, T., Grieveson, C. Multidisciplinary care for tracheostomy patients: a systematic review. Garrubba et al. Critical Care Vol 13 No 6 - Nov 2009 , p. 1-6.

Garuti, G., Reverberi, C., Briganti, A., Massobrio, M., Lombardi, F., Lusuardi, M. Swallowing disorders in tracheostomised patients: a multidisciplinary / multiprofessional approach in decannulation protocols. Garuti et al. Multidisciplinary Respiratory Medicine 2014, 9:36.

Ibrahim, S. G., Silva, J. M., Borge, L. G. A., Savi, A., et al. Utilização de equipamentos de ventilação não invasiva na traqueostomia: uma alternativa para alta da UTI. Rev Bras Ter Intensiva. 2012; 24 (2): 167-172.

Jung, Yooun-joong et al. The effect of systematic approach to tracheostomy care in patients transferred from the surgical intensive care unit to general ward. Acute and Critical Care, 30 nov. 2018. Disponível em: 22 set. 2019.

Kutsukutsa, J., Kuupiel, D., Monori-Kiss, A., Del Rey-Puech, P., Mashamba-Thompson, T. P. Tracheostomy decannulation methods and procedures for assessing readiness for decannulation in adults: a systematic scoping review. Int J Evid Based Healthc. 2019 May 31. doi: 10.1097/XEB.0000000000000166. Epub ahead of print. PMID: 31162271.

Lemos, M. M., Bandeira, C. O. P., Marques, V. D., Teixeira, J. J. V., Carvalho, M. D. B. Laringotraqueoscopia flexível na decanulação de pacientes traqueostomizados: otimização de segurança para o paciente. Saúde e Pesqui. 2019 maio-ago; 12(2): 377-383 - e-ISSN 2176-9206-9206.

Mallmann, L. P. Manejo do paciente traqueostomizado e decanulação - o papel do intensivista além da UTI. Revista Interdisciplinar do Pensamento Científico. ISSN: 2446-6778 N 2, volume 5, artigo nº 09, Julho/Dezembro 2019. D.O.I: http://dx.doi.org/10.20951/2446-6778/v5n2a9

Mandaville, A., Ray, A., Robertson, H., Foster, C., Jesser, C. A retrospective review of swallow dysfunction in patients with severe traumatic brain injury. Dysphagia 2014, [Epub ahead of print].

Mangilli, L. D., Moraes, D. P., Medeiros, G. C. Protocolo de avaliação fonoaudiológica preliminar. In: Andrade CRF, Limongi SCO (Org). Disfagia: prática baseada em evidências. São Paulo: Sarvier; 2012. p. 45-61.

Medeiros, G. C., Sassi, F. C., Silva, C. L., Andrade, C. R. F. Criteria for tracheostomy decannulation: literature review. CoDAS 2019;31(6):e20180228 DOI: $10.1590 / 2317-1782 / 20192018228$.

Melgaço, S. A., Vicente, L. C. C., Gama, A. C. C. Análise do tempo de decanulação e liberação de via oral em pacientes com câncer de boca. CoDAS 2021;33(4):e20190236 DOI: 10.1590/2317-1782/20202019236.

Nelson, J. E., Cox, C. E., Hope, A. A., Carson, S.S. Chonic critical illness. Am J Respir Crit Care Med. $2010 ; 182$ (4): $446-54$.

Padovani, A. R., Moraes, D. P., Sassi, F. C., Andrade, C. R. F. Avaliação clínica da deglutição em unidade de terapia intensiva. CoDAS. São Paulo, 2013; 25(1) p.1-7.

Penitenti, R. M., et al. Controle da pressão do cuff na unidade terapia intensiva: efeitos do treinamento. Rev Bras Ter Intensiva. 2010 ; 22(2):192-195. 
Research, Society and Development, v. 10, n. 13, e559101321517, 2021

(CC BY 4.0) | ISSN 2525-3409 | DOI: http://dx.doi.org/10.33448/rsd-v10i13.21517

Presto, B., Damázio, L. Fisioterapia na UTI. 2a ed. Rio de Janeiro: Elsevier, 2009. p. 185-195.

Santana, L., Fernandes, A., Brasileiro, Â. G., Abreu, A. C. Critérios para avaliação clínica fonoaudiológica do paciente traqueostomizado no leito hospitalar e internamento domiciliar. Rev. CEFAC. 2014 Mar-Abr; 16(2):524-536.

Simão, M. A., Alacid, C. A. N., Rodrigues, K. A., Albuquerque, C., Furkim, A. M. Incidence of tracheal aspiration in tracheotomized patients in use of mechanical ventilation. Arq Gastroenterol v. 46 - no.4 - out./dez. 2009, p. 311-314.

Vianna, A., Palazzo, R. F., Aragon, C. Traqueostomia: uma revisão atualizada. Pulmão RJ 2011; 20(3):39-42.

Zanata, I. L., Santos, R. S., Marques, J. M., Hirata, G. C., Santos, D. A. Avaliação fonoaudiológica para decanulação traqueal em pacientes acometidos por traumatismo cranioencefálico. Rev. CoDAS, 2016;28(6):710-716. 